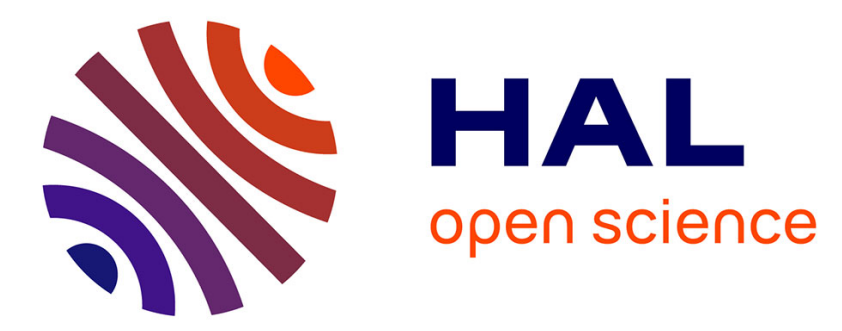

\title{
Spark Plasma Sintering à partir de poudres mécaniquement activées : compréhension des transitions de phase au cours d'un frittage réactif
}

\author{
G. Cabouro, N. Reinfried, Eric Gaffet, Y. Grin, S. Chevalier, F. Bernard
}

\section{To cite this version:}

G. Cabouro, N. Reinfried, Eric Gaffet, Y. Grin, S. Chevalier, et al.. Spark Plasma Sintering à partir de poudres mécaniquement activées: compréhension des transitions de phase au cours d'un frittage réactif. Matériaux \& Techniques, 2007, 95 (4-5), pp.269-280. 10.1051/mattech:2008011 . hal-00599067

\section{HAL Id: hal-00599067 https://hal.science/hal-00599067}

Submitted on 8 Jun 2011

HAL is a multi-disciplinary open access archive for the deposit and dissemination of scientific research documents, whether they are published or not. The documents may come from teaching and research institutions in France or abroad, or from public or private research centers.
L'archive ouverte pluridisciplinaire HAL, est destinée au dépôt et à la diffusion de documents scientifiques de niveau recherche, publiés ou non, émanant des établissements d'enseignement et de recherche français ou étrangers, des laboratoires publics ou privés. 


\title{
Spark Plasma Sintering à partir de poudres mécaniquement activées : compréhension des transitions de phase au cours d'un frittage réactif
}

\author{
G. Cabouro' ${ }^{1}$, N. Reinfried ${ }^{2}$, E. Gaffet ${ }^{3}$, Y. Grin ${ }^{2}$, S. Chevalier ${ }^{1}$ \\ et F. Bernard ${ }^{1}$ \\ 1 Institut Carnot de Bourgogne, UMR 5209 - CNRS, Université de Bourgogne, 9 avenue Alain Savary, \\ BP. 47870, 21078 Dijon Cedex, France \\ e-mail : gcabouro@u-bourgogne.fr \\ 2 Nanomaterials Research Group (NRG), UMR 5060 - CNRS, Université de Technologie de Belfort- \\ Montbéliard (UTBM), Site de Sévenans, 90010 Belfort Cedex, France \\ e-mail : Eric.Gaffet@utbm.fr \\ 3 Max-Planck-Institute for Chemical Physics of Solids, Nöthnitzer Str. 40, 01187 Dresden, Germany \\ e-mail : grin@cpfs.mpg.de
}

Mots-clés :

$\mathrm{MoSi}_{2}$; broyage; Spark Plasma Sintering; Self Propagating High Temperature Synthesis; transitions de phase

\section{Key words:}

$\mathrm{MoSi}_{2}$; mechanical milling; Spark Plasma Sintering; Self Propagating High Temperature Synthesis; phase transitions
Résumé - À « basse température " (entre 400 et $600{ }^{\circ} \mathrm{C}$ ), l'oxydation de $\mathrm{MoSi}_{2}$ entraîne sa désintégration en poudre (phénomène de "peste »). De récents travaux ont montré que l'utilisation de $\mathrm{MoSi}_{2}$ dense et nano-organisé permettrait de ralentir ce phénomène de " peste ". Le défi de produire des matériaux denses et nano-organisés peut être relevé par le frittage "flash » réactif sous champ électrique à partir des poudres mécaniquement activées (Mechanically-Activated Spark Plasma Sintering, MASPS). Le contrôle de la composition et de la microstructure du composé intermétallique $\mathrm{MoSi}_{2}$ nécessite de déterminer les paramètres du frittage SPS (température, rampe de montée en température, pression uniaxiale appliquée et temps de maintien) lorsque des poudres activées mécaniquement sont utilisées. À l'heure actuelle, par ce procédé, les échantillons présentent une phase majoritaire, $\mathrm{MoSi}_{2}$ à plus de $97 \%$, et une phase secondaire, $\mathrm{Mo}_{5} \mathrm{Si}_{3}$. Il est donc indispensable de comprendre les transitions de phase qui peuvent avoir lieu au cours d'un frittage impliquant simultanément une consolidation et une réaction SHS. Il a donc été essentiel de développer un nouveau type d'équipement pour reproduire partiellement ce procédé. Ceci a été réalisé avec succès en mettant en œuvre une analyse par diffraction des rayons $X$ « in-situ » et en temps réel couplée à une analyse par thermographie IR.

\begin{abstract}
Spark Plasma Sintering from mechanically-activated powders: In-situ investigation of phase transition during a current activated SHS. At "low temperature" (between 400 and $600^{\circ} \mathrm{C}$ ), an accelerated oxidation can be occurred enhancing the disintegration of the massive $\mathrm{MoSi}_{2}$. This phenomenon was named "pest oxidation". It is generally allowed that the "pest" phenomenon, i.e. powder disintegration of massive $\mathrm{MoSi}_{2}$ samples, is due to the growth of $\mathrm{Mo}$ oxide $\left(\mathrm{MoO}_{3}\right)$. Recents works have shown that the use of dense nano-organized $\mathrm{MoSi}_{2}$ can be slowed down this phenomenon. The challenge for producing such dense nanostructured materials has been reached from a mechanically-activated spark plasma sintering (MASPS). In order to synthesize a dense molybdenum disilicide with a perfectly controlled microstructure, an investigation of the influence of Spark Plasma Sintering processing parameters (temperature, heating rate, mechanical pressure and holding time) on the chemical composition and the microstructure characteristics has been performed. Such an approach leads to the elaboration of samples getting a porosity of $6 \%$ and a microstructure nano-organized. But these optimal conditions did not make it possible to really obtain the expected phase compound during the reactive sintering of $\mathrm{MoSi}_{2}$. Indeed, both XRD analysis and SEM observation reveal the presence of a secondary phase, $\mathrm{Mo}_{5} \mathrm{Si}_{3}$. Consequently, it was essential to develop characterization tools "in-situ" such as the Time Resolved X-ray Diffraction (TRXRD), with an X-ray synchrotron beam (D2AM-BM2, ESRF Grenoble) coupled to an infrared thermography to study simultaneously structural transformations and thermal evolutions. In situ synchrotron time-resolved X-ray diffraction experiments coupled with an infrared imaging camera have been used to reveal the reaction mechanisms during the production of $\mathrm{MoSi}_{2}$ by a current activated SHS sintering.
\end{abstract}

e disiliciure de molybdène, $\mathrm{MoSi}_{2}$, est un matériau prometteur pour des applications à très haute température. Cet intermétallique possède un haut point de fusion $\left(2030{ }^{\circ} \mathrm{C}\right)$, une bonne conductivité électrique 
et allie une bonne résistance au fluage et à l'oxydation [1]. À l'heure actuelle, ce matériau est principalement utilisé comme élément chauffant pour des fours à très haute température, mais également comme matériau de revêtement sur du molybdène par exemple. Plusieurs procédés ont été développés pour produire cet intermétallique avec une microstructure fine, comme, par exemple, des réactions à l'état solide [2]. Le recours aux techniques impliquant une étape de broyage constitue une solution prometteuse pour obtenir des matériaux à microstructure parfaitement contrôlée, soit de manière directe (mécano synthèse $[3,4]$ ), ou indirecte après un traitement thermique des poudres mécaniquement activées. En effet, l'enthalpie de formation de ce matériau $\left(\Delta H_{\mathrm{f}}=\right.$

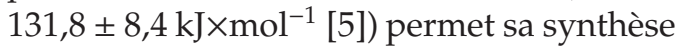
par des réactions auto-entretenues ou SelfPropagating High-Temperature Synthesis (SHS) à partir d'un mélange stoechiométrique de poudres activées mécaniquement de Mo et de Si, pré-compacté à froid. Ce cru est chauffé d'un côté, une réaction est initiée dans un faible volume. Une fois la réaction initiée, le front de propagation, qui peut atteindre des vitesses allant de 700 à $3300{ }^{\circ} \mathrm{C} \mathrm{s}^{-1}$, traverse la totalité de l'échantillon, entraînant la formation du composé désiré. De plus, ce procédé permet de produire des échantillons massifs avec un temps de frittage beaucoup plus court que par les autres procédés de la métallurgie des poudres [6-8].

Pour obtenir des matériaux denses, il est nécessaire de rajouter une étape de consolidation à ce procédé [9]. Des études récentes ont montré toute la pertinence de l'utilisation d'un courant électrique de forte intensité à basse tension assisté d'une pression pour synthétiser et consolider des matériaux à microstructure nano-organisée en une seule étape dans de nombreux systèmes (FeAl [10], $\mathrm{MoSi}_{2}$ [11], $\mathrm{NbAl}_{3}$ [12]). Ce procédé, appelé MASPS (MechanicallyActivated Spark Plasma Sintering) permet l'élaboration de composés uniformément denses en un temps très court facilitant l'obtention de nanostructure. Ce procédé est composé de deux étapes principales :

(i) l'activation mécanique pour produire des agglomérats micrométriques composés de nanocristallites de Mo et de Si [10], (ii) la synthèse simultanée via des réactions auto-entretenues et la consolidation grâce au procédé SPS [10,13-15].

L'objectif de ce travail est de déterminer les conditions de synthèse SPS (température, rampe de montée en température, pression et temps de maintien) pour produire des composés $\mathrm{MoSi}_{2}$ denses, ayant une composition et une microstructure parfaitement contrôlées. Il est également nécessaire d'étudier le ou les chemin(s) réactionnel(s), en particulier les différentes transitions de phase qui peuvent avoir lieu au cours d'un frittage "flash» réactif initié par un courant électrique. Toutefois, en raison des conditions extrêmes associées aux réactions SHS, l'utilisation d'outils de caractérisations adaptés est indispensable. Ceci est maintenant possible grâce aux équipements développés au LURE (Laboratoire d'Utilisation du Rayonnement Électromagnétique), voire plus récemment à l'ESRF (European Synchrotron Radiation Facility) pour suivre en temps réel et de manière «in-situ» les évolutions structurales et thermiques qui ont lieu au cours de ces réactions. Ainsi, une identification des mécanismes réactionnels, en vue de maîtriser les étapes déterminantes, est alors envisageable. Enfin, dans le cadre des applications structurales à haute température, il est très important d'étudier le comportement de notre matériau à «basse température » (entre 400 et $600{ }^{\circ} \mathrm{C}$ ) sous air. En effet, dans cette gamme de température, l'oxydation de $\mathrm{MoSi}_{2}$ est accélérée, ce qui entraîne la désintégration du matériau en poudre [16]. Récemment, Chevalier et al. [17] ont montré que le phénomène de "peste » est plus prononcé suite à l'oxydation sous air à $400{ }^{\circ} \mathrm{C}$ de composés $\mathrm{MoSi}_{2}$ élaborés par des réactions $\mathrm{SHS}$ à partir d'un mélange de poudres mécaniquement activées. Cette température d'oxydation a donc été choisie.

\section{Frittage " flash " à partir de mélanges de poudres mécaniquement activés}

\section{Activation mécanique}

Un mélange stoechiométrique de Mo (Sigma-Aldrich, - 100 mesh) et de Si (SigmaAldrich, - 325 mesh) a été broyé à l'aide d'un broyeur planétaire Fritsch (vario-mill P4 Pulverisette) [18], qui permet de sélectionner indépendamment la fréquence de choc et l'énergie de choc en jouant sur les 


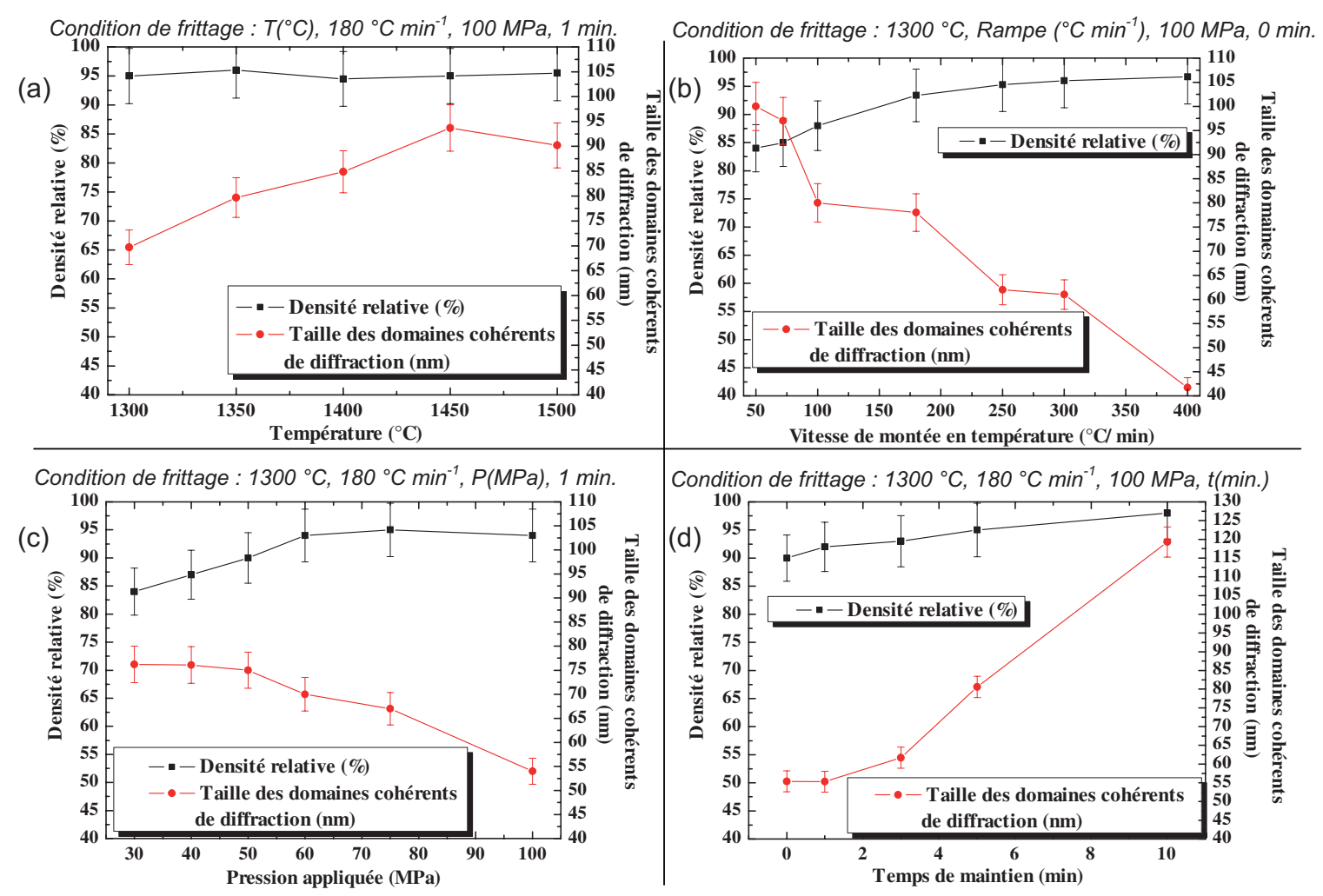

Fig. 1. Effet des différents paramètres de frittage sur la densité relative et sur la taille des domaines cohérents de diffraction X sur les produits frittés par MASPS.

Fig. 1. Influence of processing parameters on the relative density and on the coherently diffracting domain size of products elaborated by MASPS.

vitesses du plateau par rapport à celles des jarres. À partir d'une étude récente [13], les conditions de broyage ont été fixées comme suit : 350 RPM (Rotation Par Minute) pour le plateau, -250 RPM pour les jarres et un temps de broyage de $2 \mathrm{~h}$. Ainsi, ce type de broyage permet d'obtenir des agglomérats micrométriques (entre 0,2 et $500 \mu \mathrm{m}$ ) composés de nanocristallites de Mo $(50 \mathrm{~nm})$ et de $\mathrm{Si}(20 \mathrm{~nm})$.

\section{Synthèse SPS de MoSi 2}

Le mélange activé mécaniquement est pressé uniaxialement $(80 \mathrm{MPa})$ dans une matrice en carbone. Ce compact, ayant une densité relative de $60 \%$, est ensuite soumis simultanément à un courant direct $(D C)$ pulsé de forte intensité et à une pression uniaxiale. Comme la matrice en carbone est chauffée par le courant la traversant, la poudre est chauffée à la fois de l'intérieur mais également de l'extérieur par effet Joule. Après un court temps de maintien, le courant est coupé [19].

Shen et al. $[20,21]$ ont démontré qu'il est possible de contrôler la microstructure (défaut, taille, etc.) des produits finaux en contrôlant les paramètres de frittage par
SPS. Dans cette étude, 4 paramètres ont été considérés (la température, la rampe de montée en température, la pression et le temps de maintien). Des corrélations entre ces différents paramètres ont été établies grâce à une mesure de la densité relative par la poussée d'Archimède. Une étude préliminaire a montré qu'il est nécessaire d'atteindre une température de frittage de $1300{ }^{\circ} \mathrm{C}$ pour élaborer un échantillon avec une densité acceptable (>85\%). Néanmoins, comme le montre la figure 1a, une augmentation de la température n'entraîne pas forcément une augmentation de la densité relative. En revanche, cette augmentation de la température entraîne une augmentation de la taille des domaines cohérents de diffraction $X$ qui passe de $65 \pm 0,2 \mathrm{~nm}$ à $90 \pm 0,2 \mathrm{~nm}$. La pression appliquée au cours du frittage, mais également le temps de maintien (Figs. 1c et 1d) ont un effet bénéfique sur l'évolution de la densité relative. Une augmentation de ces deux paramètres jusqu'à une valeur limite, qui est respectivement de $60 \mathrm{MPa}$ pour la pression appliquée et de $5 \mathrm{~min}$ pour le temps de maintien, permet une augmentation de la densité relative. Une fois ces valeurs limites 
(a)
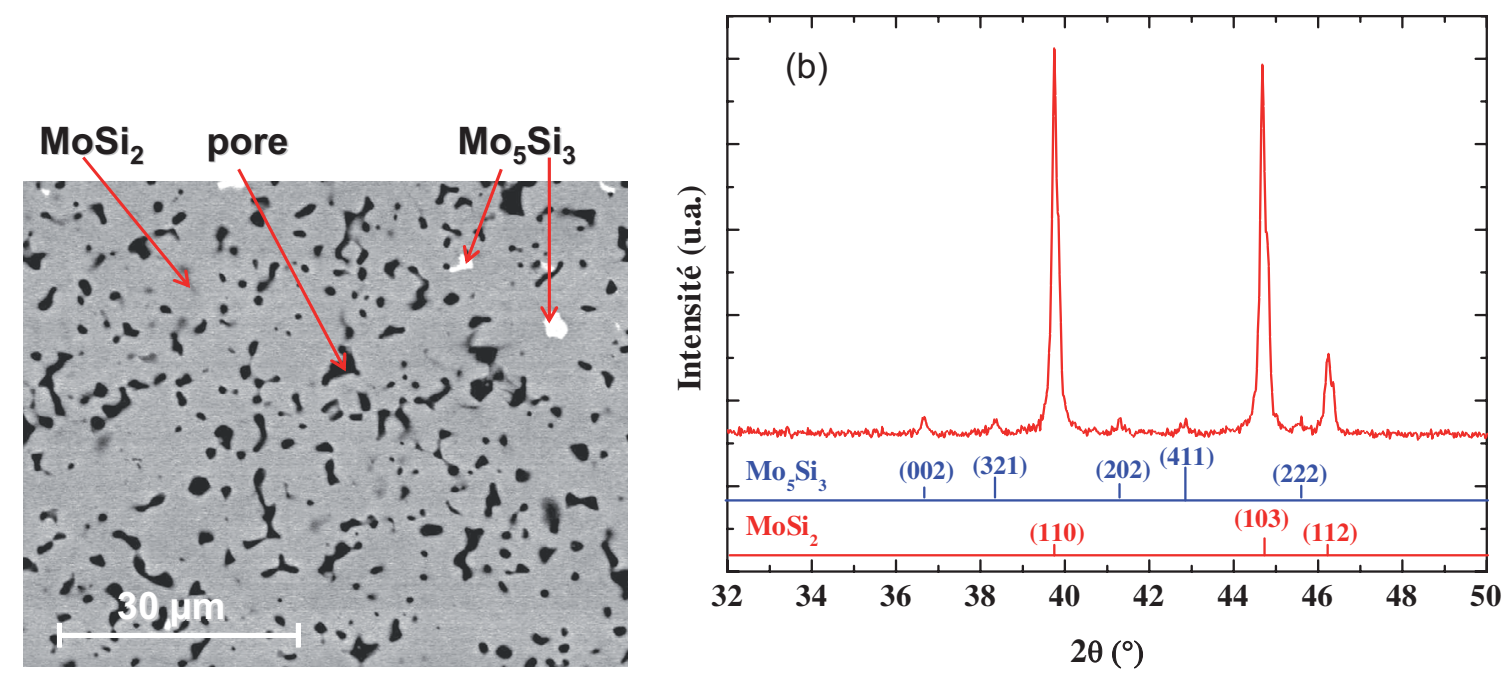

Fig. 2. Composé $\mathrm{MoSi}_{2}$ dense et nano-organisé, fritté à $T_{\mathrm{f}}(\mathrm{Si}), 400{ }^{\circ} \mathrm{C} / \mathrm{min}, 100 \mathrm{MPa}$, sans temps de maintien à partir de poudres mécaniquement activées : (a) morphologie MEB de la surface des échantillons, (b) analyses des phases par diffraction des rayons $X$.

Fig. 2. Dense and nano-organized MoSi $i_{2}$ sintered at $\mathrm{Tm}(\mathrm{Si}), 400^{\circ} \mathrm{C} / \mathrm{min}, 100 \mathrm{MPa}$ and without holding time: (a) SEM micrograph of the sample surface, (b) X-ray diffraction patterns.

atteintes, la densité n'évolue plus. Cependant, ces deux paramètres ont une influence opposée sur la taille des domaines cohérents de diffraction. En effet, alors que la pression a un effet bénéfique sur ce dernier paramètre (Fig. 1c), l'augmentation du temps de maintien entraîne une augmentation de la taille des domaines cohérents de diffraction (Fig. 1d), rendant difficile la conservation de la nanostructure. Le dernier paramètre étudié est l'effet de la rampe de montée en température (Fig. 1b). L'augmentation de ce paramètre entraîne clairement une augmentation de la densité relative, mais surtout une forte diminution de la taille des domaines cohérents de diffraction (Fig. 1b) qui passe de $93 \pm 0,2 \mathrm{~nm}$ à $41 \pm 0,2 \mathrm{~nm}$.

Le contrôle des paramètres de frittage permet d'élaborer des échantillons denses à plus de $96 \%$ et présentant une nanoorganisation. En effet, la taille des domaines cohérents de diffractions $X$ dans des échantillons élaborés en employant les conditions optimales $\left(1300{ }^{\circ} \mathrm{C}, 400^{\circ} \mathrm{C} \times \mathrm{min}^{-1}, 100 \mathrm{MPa}\right.$, sans temps de maintien) est comprise entre $41 \pm 0,2$ et $45 \pm 0,2 \mathrm{~nm}$. Néanmoins, ces conditions ne permettent pas d'obtenir un échantillon monophasé. En effet, comme le montrent les figures $2 a$ et $2 b$, les échantillons frittés par frittage «flash » réactif présentent deux phases :

(i) $\mathrm{MoSi}_{2}$ (phase majoritaire, à 96-97\% en masse),

(ii) $\mathrm{Mo}_{5} \mathrm{Si}_{3}$ (phase secondaire, à 3-4 \% en masse).
Une analyse des courbes de l'évolution du retrait de l'échantillon et de l'intensité du courant électrique en fonction du temps [22] a montré que cette phase secondaire se formait majoritairement au cours du préchauffage de l'échantillon, c'est-à-dire avant la température de fusion du silicium.

\section{Investigation " in-situ " des mécanismes réactionnels conduisant à la formation du composé $\mathrm{MoSi}_{2}$ au cours d'un frittage « flash " réactif initié par un courant électrique}

\section{Description du montage}

La réaction SHS $\left(\mathrm{Mo}+2 \mathrm{Si} \rightarrow \mathrm{MoSi}_{2}\right)$ qui a lieu au cours du frittage par SPS a été étudiée "in-situ » et en temps réel par diffraction des rayons X (TRXRD, Time Resolved X-ray diffraction, Fig. 3a) en utilisant $d^{\prime}$ 'une part le rayonnement $X$ d'un synchrotron (ligne D2AM-BM2, ESRF, Grenoble) et d'un détecteur courbe (INEL) ayant une ouverture angulaire de $80^{\circ}$ et, d'autre part, une caméra IR (AVIO TVS 2000ST) pour enregistrer les évolutions thermiques à la surface de l'échantillon. De plus, pour reproduire les conditions de ce frittage "flash» par SPS, un nouveau porte-échantillon a été mis en oeuvre pour reproduire le plus fidèlement possible le mode d'ignition du procédé de frittage «flash » réactif sous champ électrique, en permettant le passage d'un 

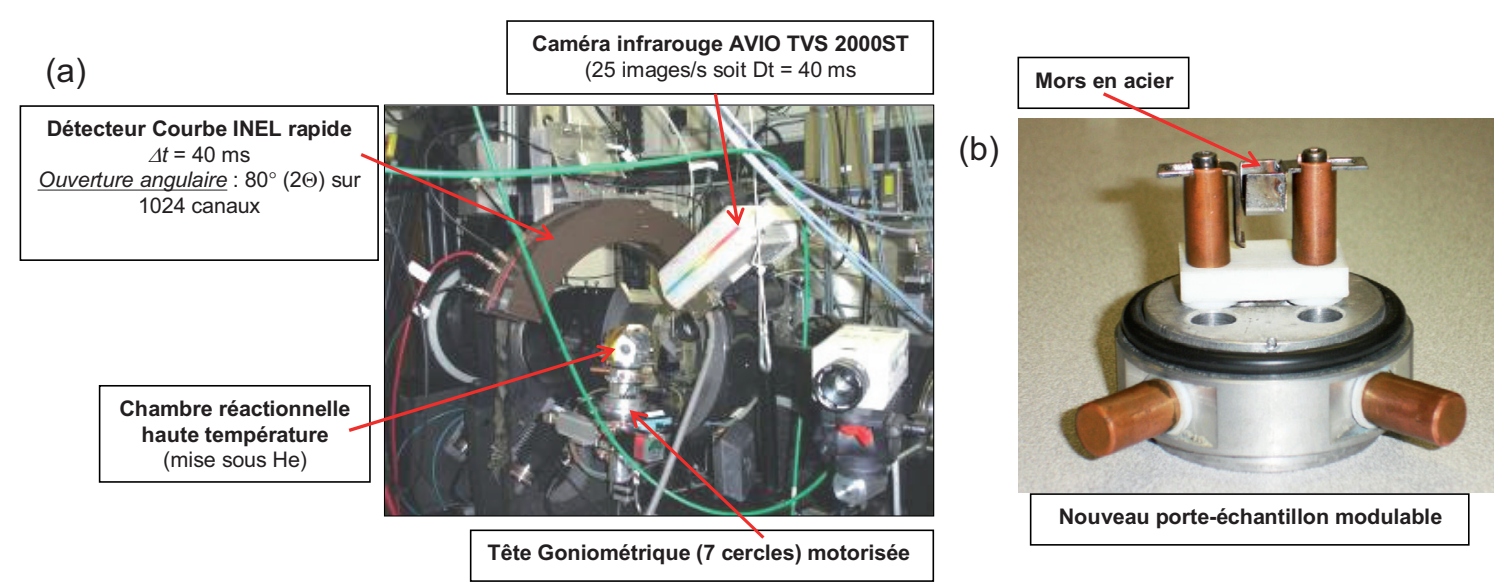

Fig. 3. (a) Dispositif expérimental TRXRD - IR utilisé sur la ligne de lumière D2AM-BM2 (ESRF), (b) photographie du nouveau porte-échantillon réalisé pour la campagne de mesure à l'ESRF.

Fig. 3. (a) General description of TRXRD device used on the D2AM-BM2 beamline, (b) description of the sample holder allowing an electrical current within the sample.

courant au sein de l'échantillon (Fig. 3b). Les réactions SHS sont initiées par un courant électrique alternatif $(A C)$, qui est délivré par un générateur permettant le passage d'un courant de forte intensité. Récemment, Chen et al. [23] ont rapporté que l'épaisseur de la couche de $\mathrm{MoSi}_{2}$ formée est indépendante du courant utilisé pour l'ignition.

La longueur d'onde sélectionnée est fixée à $0,177 \mathrm{~nm}$, dans le but de séparer au mieux les pics de diffraction. De plus, une paire de filtres a été utilisée pour minimiser la taille de spot de rayons $X$ sur l'échantillon dans la direction perpendiculaire au front de combustion. Le faisceau de rayons $X$ du synchrotron frappe l'échantillon, qui est placé à l'intérieur d'une chambre réactionnelle haute température, équipée d'une fenêtre de Kapton (ouverture de $190^{\circ}$ ) parallèle au détecteur courbe INEL (CPS 120) (Fig. 3a). Le temps d'acquisition entre deux diffractogrammes consécutifs est égal à $40 \mathrm{~ms}$. Cette chambre réactionnelle, qui est sous atmosphère d'hélium à la pression atmosphérique, est placée sur un goniomètre 7 cercles avec une précision de $0,001^{\circ}$ et un ajustement vertical. La caméra infrarouge est équipée d'une lentille permettant d'obtenir une image de $9,5 \mathrm{~cm} \times 6,25 \mathrm{~cm}$ et chaque pixel de chaque image infrarouge correspond à une aire de $0,79 \mathrm{~mm} \times 0,79 \mathrm{~mm}$.

\section{Mécanisme réactionnel du frittage " flash » réactif : TRXDR couplée avec IR}

La caméra infrarouge enregistre l'évolution thermique de l'échantillon au cours de la réaction initiée par le passage du courant.
Le temps de réaction est faible, puisque le front de propagation traverse l'échantillon en moins de $6 \mathrm{~s}$. L'étude des données IR permet d'accéder à la vitesse du front mais aussi à la stabilité de ce dernier suivant l'axe de propagation du front de combustion $(C D)$ et un axe perpendiculaire à ce dernier $(A B)$ situé au milieu de l'échantillon $(x=0 \mathrm{~mm})$ (Fig. 4a). Les positions des thermogrammes infrarouges enregistrés le long de $(C D)$ pendant le passage du front de combustion pour trois positions consécutives choisies $\left(x_{1}, x_{2}\right.$, $\left.x_{3}\right)$ et le long de $(A B)$ ont été tracées (Figs. $4 \mathrm{~b}$ et 4c). L'analyse des données thermiques selon $(C D)$ montre que le front de combustion est stable et présente une vitesse moyenne de $15 \pm 1 \mathrm{~mm} \mathrm{~s}^{-1}$ (Fig. 4c). Cependant, l'analyse réalisée selon l'axe $(A B)$ montre que le front de propagation n'est pas rigoureusement perpendiculaire à l'axe de propagation de ce dernier (Fig. 4b). Le transfert thermique a lieu d'agglomérats en agglomérats [24]. Or, il est important de rappeler que les analyses structurale (TRXRD) et thermique (IR) s'effectuent sur une grande surface (respectivement $1 \times 1,5 \mathrm{~mm}^{2}$ et $0,7 \times 0,7 \mathrm{~mm}^{2}$ ) ce qui signifie que les informations obtenues dans cette zone sont moyennées. Ainsi, à chaque instant, dans la zone irradiée, il y a des agglomérats qui auront déjà réagi, d'autres qui sont en cours de réaction et ceux qui n'auront toujours pas réagi.

Les évolutions structurales au cours de ces réactions sont présentées sur la figure 5 . Sur cette figure, les premiers pics de diffraction sont enregistrés juste avant la réaction d'ignition. La fusion du silicium peut être repérée par la disparition complète du pic Si (311). Au cours de la réaction, le 
(a)

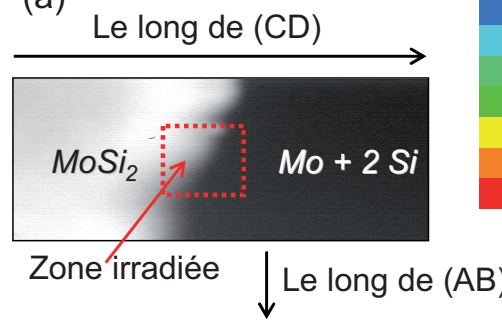

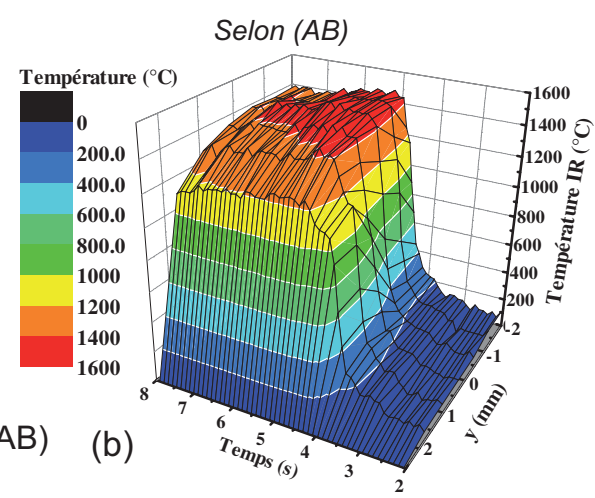

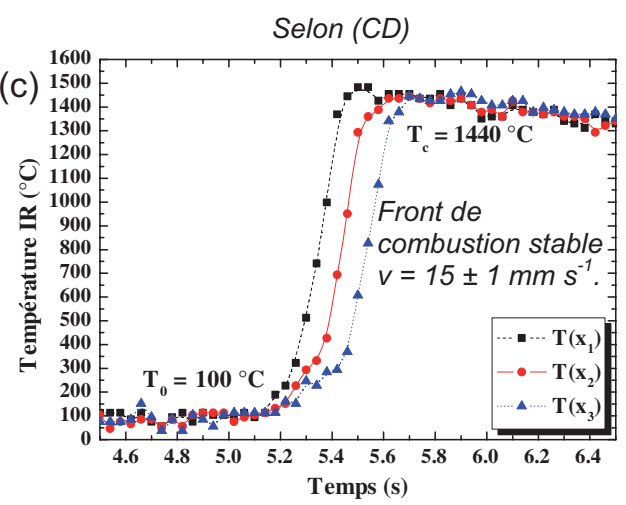

Fig. 4. Analyse thermique de la réaction de $\mathrm{Mo}+2 \mathrm{Si} \rightarrow \mathrm{MoSi}_{2}$ : (a) image macroscopique du front de propagation au cours de la réaction SHS initiée par un courant électrique, $(b)$ analyse thermique le long de l'axe $(A B)$ perpendiculaire à au front de combustion au milieu de l'échantillon, (c) trois thermogrammes enregistrés le long de l'axe $(C D)$ du front de combustion aux positions $x_{1}, x_{2}$ et $x_{3}$.

Fig. 4. Thermal analysis on a Mo +2 Si sample: (a) macroscopic micrograph of the propagation wave, (b) thermal analysis of the combustion front propagation along $(A B)$, (c) thermal analysis of the combustion front propagation along $(C D)$ for three consecutive selected area $\left(x_{1}, x_{2}, x_{3}\right)$.

(a)

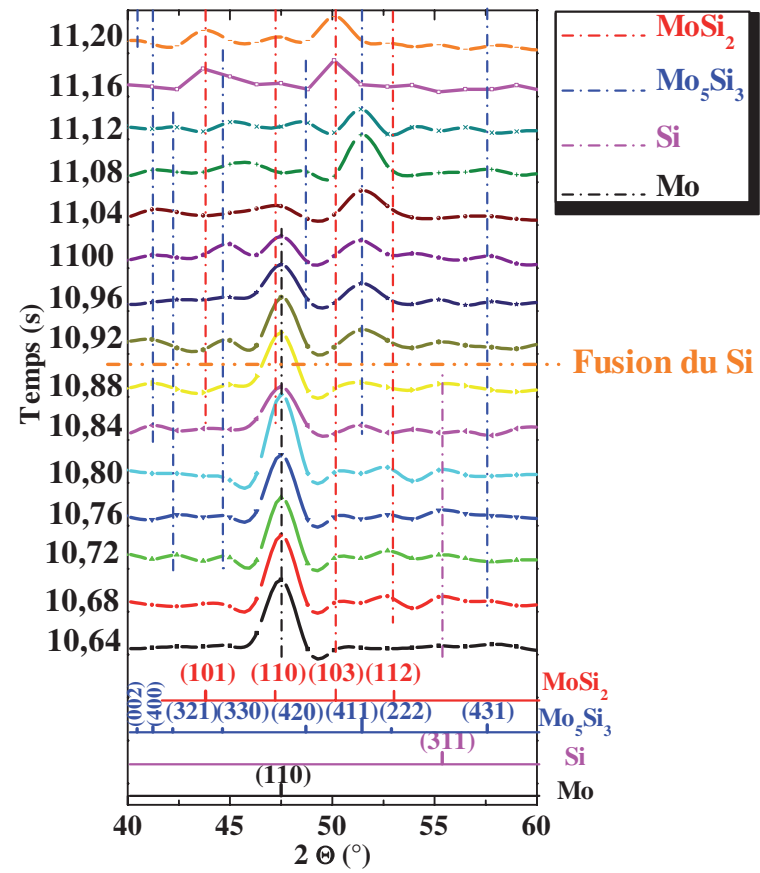

(b)
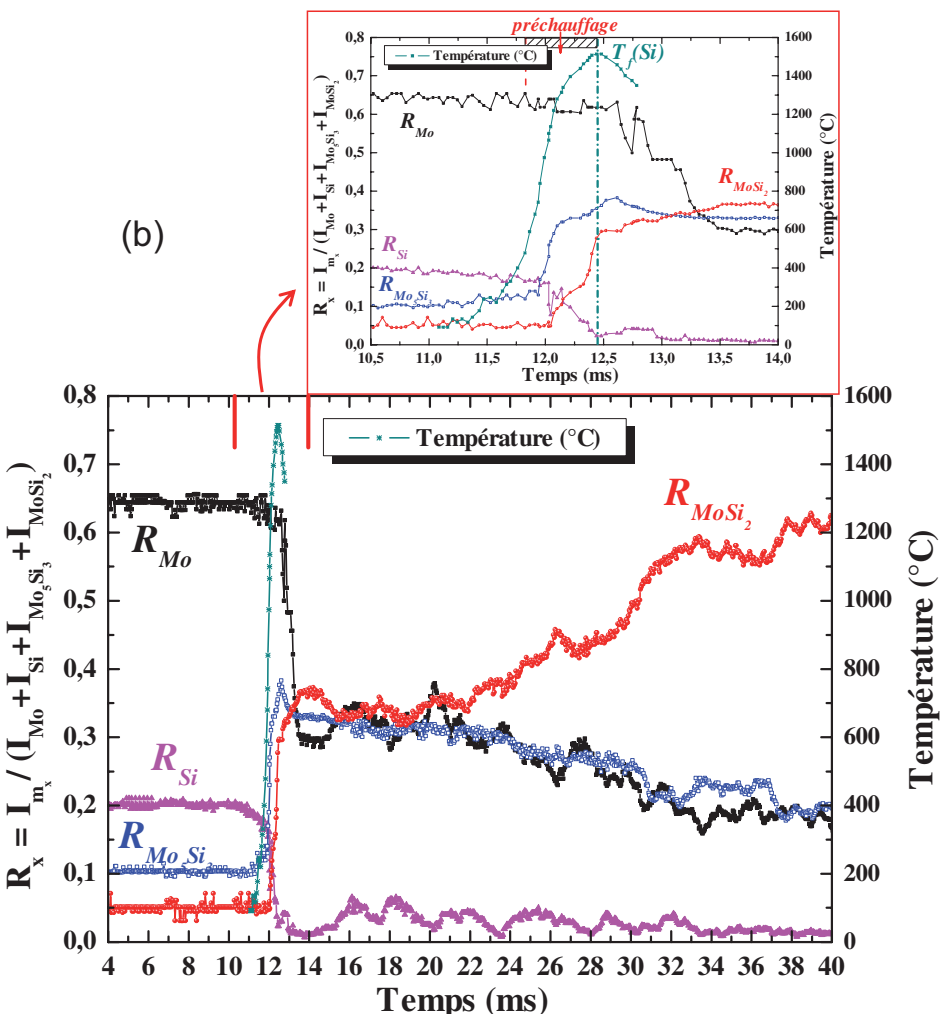

Fig. 5. Diffraction des rayons $X$ en «temps réel» : (a) sélections des diagrammes des rayons $X$ où les modifications structurales majeures ont été observées en fonction du temps à l'intérieur de la zone irradiée (au centre de l'échantillon) au cours du frittage "flash » réactif (résolution temporelle $=40 \mathrm{~ms}$ ), et $(\mathrm{b})$ évolution du taux de conversion $R_{\mathrm{x}}$ des différentes phases considérées (Mo, $\mathrm{Si}_{1}, \mathrm{Mo}_{5} \mathrm{Si}_{3}$ et $\mathrm{MoSi}_{2}$ ) en fonction du temps.

Fig. 5. Time-resolved diffractograms: (a) maximum intensity of XRD peaks (reactants and products) of each phase versus the time inside the irradiated area (at the middle of the sample) during the current-activated SHS-sintering (temporal resolution $=40 \mathrm{~ms}),(b)$ evolution of the volume fraction of each phase, $\mathrm{Rx}$ ratio, $\left(x=\mathrm{Mo}, \mathrm{Si}_{1} \mathrm{Mo}_{5} \mathrm{Si}_{3}\right.$ et MoSi $\left.i_{2}\right)$ versus time. 
rapport de l'intensité maximale de la raie sélectionnée de la phase $\mathrm{X}$ sur la somme des intensités des raies des composés présents est proportionnel au volume diffractant sans pour autant être fonction des conditions de diffraction. On introduit alors le taux de conversion $R_{\mathrm{x}}$ comme une fonction qui, en première approximation, est indicatrice des évolutions structurales en fonction du temps. Le rapport $R_{\mathrm{x}}$ pour la phase $\mathrm{X}$ a pour équation: $R_{\mathrm{x}}=I_{\mathrm{x}} /\left(I_{\mathrm{Si}}+I_{\mathrm{Mo}}+I_{\mathrm{MoSi}_{2}}+I_{\mathrm{Mo}_{5} \mathrm{Si}_{3}}\right)$, où $I_{\mathrm{x}}$ correspond à l'intensité maximale des pics enregistrés au cours des expériences de diffraction des rayons $X$ en «temps réel ». Dans le but de déterminer la fraction volumique de l'échantillon en suivant l'évolution du rapport $R_{\mathrm{x}}\left(\mathrm{x}=\mathrm{Mo}, \mathrm{Si}, \mathrm{Mo}_{5} \mathrm{Si}_{3}, \mathrm{MoSi}_{2}\right)$, les intensités des pics de $\operatorname{Mo}(110), \operatorname{Si}(311)$, $\mathrm{Mo}_{5} \mathrm{Si}_{3}(411), \mathrm{MoSi}_{2}(103)$ ont été déterminées pour calculer ce rapport (Fig. 5b).

Quel que soit le mécanisme SHS, une interface réactive entre les particules de Mo mouillées par le silicium liquide est considérée. Dans la littérature, deux scénarii ont été développés :

(i) un mécanisme de diffusion-réactive, qui considère la formation d'une couche de $\mathrm{MoSi}_{2}$ sur la surface des particules de Mo par diffusion du Si liquide,

(ii) un mécanisme de dissolution-précipitation, basé sur une dissolution des particules de Mo dans le Si liquide qui est suivie d'une précipitation de $\mathrm{MoSi}_{2}$.

Néanmoins, ces deux mécanismes ne peuvent expliquer totalement les transitions de phase qui ont lieu au cours du frittage «flash" réactif de $\mathrm{MoSi}_{2}$. En effet, les auteurs de ces mécanismes utilisent une source de chaleur extérieure pour amorcer la réaction SHS. De plus, le courant appliqué, qui traverse l'échantillon, peut avoir une influence non négligeable sur les mécanismes de formation des phases et par voie de conséquence sur la nature et la microstructure du produit final. En effet, le courant électrique appliqué peut influencer le processus de frittage « flash » réactif par :

(i) effet Joule,

(ii) une amélioration du transport de masse due à l'électromigration ou à la génération de défauts qui sont nombreux dans les poudres broyées, ou

(iii) la création d'une décharge électrique (ou/et d'un plasma) entre les particules $[25,26]$.

Gao et al. [27], utilisant des trempes au cours du procédé, suggèrent que le mécanisme de formation de $\mathrm{MoSi}_{2}$ au cours du procédé SHS n'est ni un mécanisme de diffusionréactive, ni un mécanisme de dissolutionprécipitation. Ce mécanisme est similaire au mécanisme de formation de TiC proposé par Alexandrov et al. [28] et de NiAl proposé par Biswas et Roy [29]. Néanmoins, le mécanisme suggéré par Gao et al. [27] n'inclut pas de réaction à l'état solide au cours du préchauffage.

Avant le déclenchement de la réaction SHS, la présence d'une faible quantité de $\mathrm{MoSi}_{2}$ (fiche ICDD No. 41-0612) produite lors de l'étape d'activation mécanique peut être observée. Cependant, cette phase, lorsqu'elle est en faible quantité ( $<5 \%$ en masse), a un effet positif sur la microstructure finale, puisque cette phase est un site de nucléation hétérogène permettant une diminution de la température de combustion [13].

Durant la période de préchauffage, le taux de réaction augmente. Comme le montre la figure $5 \mathrm{~b}$, entre 500 et $1000{ }^{\circ} \mathrm{C}$, la présence de la phase intermédiaire, $\mathrm{Mo}_{5} \mathrm{Si}_{3}$, peut être détectée entraînant une fluctuation du front de combustion. Deevi [30] a montré que la conduction est le premier effet du transfert thermique. Ainsi, une réaction exothermique de diffusion à l'état solide (Eq. (1)) entre le Mo solide et le Si solide a lieu dans la zone de chauffage.

$$
\mathrm{Si}(\mathrm{s})|\mathrm{Mo}(\mathrm{s}) \rightarrow \mathrm{Si}(\mathrm{s})| \mathrm{Mo}_{5} \mathrm{Si}_{3}(\mathrm{~s}) \mid \mathrm{Mo}(\mathrm{s}) .
$$

Entre $1000^{\circ} \mathrm{C}$ et la température de fusion du silicium, le taux de conversion de $\mathrm{MoSi}_{2}$ augmente (Fig. $5 b)$. Une réaction de diffusion à l'état solide entre Si solide et Mo solide pourrait se produire au cours de cette phase de préchauffage avant la fusion du silicium. Or, il est généralement admis que la formation du composé $\mathrm{MoSi}_{2}$ est due à une réaction solide-liquide entre le molybdène solide et le silicium liquide. Ainsi, une hypothèse peut être avancée pour expliquer la présence de ce composé avant le point de fusion du silicium, à savoir la présence d'une fusion partielle et localisée du Si dans quelques agglomérats activés mécaniquement, causée par la structure nanométrique du Si. Deevi [30] a montré la grande mobilité du Si liquide (environ $10^{3}-10^{5}$ plus grande que dans sa forme solide [31]). Ainsi En conséquence, le silicium liquide peut «mouiller » et enrichir $\mathrm{Mo}_{5} \mathrm{Si}_{3}$ en Si pour former $\mathrm{MoSi}_{2}$ (Eq. (2) donnée par Deevi [30]). Néanmoins, la présence de ce produit, avant le passage du front de propagation peut induire une diminution $\mathrm{du}$ gradient thermique dans l'échantillon, due à 
une dissipation de l'énergie thermique.

$$
\begin{aligned}
& \mathrm{Si}(\mathrm{l})\left|\mathrm{Mo}_{5} \mathrm{Si}_{3}(\mathrm{~s})\right| \mathrm{Mo}(\mathrm{s}) \rightarrow \\
& \mathrm{Mo}_{5} \mathrm{Si}_{3}(\mathrm{~s})+\mathrm{MoSi}_{2}(\mathrm{~s}) .
\end{aligned}
$$

Après la fusion du Si, la présence de Mo, $\mathrm{Mo}_{5} \mathrm{Si}_{3}, \mathrm{MoSi}_{2}$ est confirmée sur la figure $5 \mathrm{a}$. Quand le silicium fondu mouille les particules de Mo, les atomes de silicium commencent à se diffuser dans les particules de $\mathrm{Mo}$, formant la phase intermédiaire $\mathrm{Mo}_{5} \mathrm{Si}_{3}$. En effet, après la fusion du silicium, des pics de diffraction $\mathrm{X}$ de $\mathrm{Mo}$, des phases $\mathrm{Mo}_{5} \mathrm{Si}_{3}$ et $\mathrm{MoSi}_{2}$ ont été détectées (Figs. $5 \mathrm{a}$ et $5 \mathrm{~b}$ ). $\mathrm{La}$ fraction volumique de $\mathrm{Mo}_{5} \mathrm{Si}_{3}$ diminue tandis que le taux de conversion de $\mathrm{MoSi}_{2}$ augmente. Ces évolutions semblent résulter de plusieurs réactions différentes :

(i) entre le silicium liquide et $\mathrm{Mo}_{5} \mathrm{Si}_{3}$ solide menant à $\mathrm{MoSi}_{2}$ [24],

(ii) entre Si liquide et Mo menant à $\mathrm{Mo}_{5} \mathrm{Si}_{3}$.

Cette dernière réaction peut être détectée sur le diagramme de la figure $5 \mathrm{~b}$ par une augmentation du taux de conversion de $\mathrm{Mo}_{5} \mathrm{Si}_{3}$ après la fusion du silicium. Après que le taux de silicium ait atteint sa concentration d'équilibre dans les particules de Mo, la couche de phase intermédiaire commence à se casser, en raison de la dilation thermique des particules de Mo n'ayant pas encore réagi $\left(6,6 \times 10^{-6}{ }^{\circ} \mathrm{C}^{-1}\right)$. Puis, cette phase intermédiaire commence à se dissoudre dans le silicium fondu. Cette dissolution peut être détectée par une diminution de la fraction volumique de $\mathrm{Mo}_{5} \mathrm{Si}_{3}$ (Fig. 5b). Par voie de conséquence, le silicium fondu se sature de molybdène, causant une précipitation continue de $\mathrm{MoSi}_{2}$ jusqu'à ce que les réactifs deviennent complètement consommés (Eq. (3)).

$$
\mathrm{Si}(\mathrm{l}) \mid \mathrm{Mo}(\mathrm{s}) \rightarrow \mathrm{MoSi}_{2}(\mathrm{~s}) .
$$

Les différents appareillages ont été configurés pour étudier la première minute du refroidissement de l'échantillon. Après la réaction, aucune nouvelle phase n'est apparue. Enfin, des études complémentaires semblent indiquer qu'une augmentation de la montée en température lors du frittage permettrait de réduire considérablement la proportion de $\mathrm{Mo}_{5} \mathrm{Si}_{3}$ [22].

Le mécanisme de formation du composé $\mathrm{MoSi}_{2}$ élaboré par frittage "flash » réactif sous champ électrique établi au cours de cette étude est donc similaire au mécanisme proposé par Alexandrov et al. [28] ou Biswas et Roy [29] en ajoutant une étape de préchauffage entre $500{ }^{\circ} \mathrm{C}$ et le point de fusion du silicium. Ainsi, cette nouvelle approche du mécanisme de formation est résumée sur la figure 6 .

\section{Premiers tests de corrosion}

L'étude de la résistance à l'oxydation à haute température a été réalisée en condition isotherme durant $24 \mathrm{~h}$ à $400^{\circ} \mathrm{C}$ sous air à l'aide d'une thermobalance setaram TG 92. Cette microbalance permet de mesurer une variation relative de masse de l'ordre du microgramme sous la pression atmosphérique ou sous un flux de gaz à débit contrôlé, au cours du chauffage des échantillons. Cette approche cinétique permet d'estimer la «vitesse de corrosion » du composé à la suite de l'oxydation. Une courbe d'avancement d'allure parabolique ou loi parabolique signifie que la vitesse de réaction de l'oxydation suit une loi de type :

$$
\left(\frac{\Delta m}{S}\right)^{2}=k_{\mathrm{p}} t+c t e
$$

où $\Delta m / S$ est la prise de masse par unité de surface en $\mathrm{mg} \times \mathrm{cm}^{-2}, t$ est le temps d'oxydation en $s$ et $k_{\mathrm{p}}$ est la constante de cinétique parabolique d'oxydation en $\mathrm{g}^{2} \times \mathrm{cm}^{-4} \times \mathrm{s}^{-1}$.

Ce type de loi implique que la diffusion des espèces au sein d'une couche dense et couvrante au cours de la croissance est l'étape déterminante d'un phénomène d'oxydation [32]. De plus, des oxydations de longue durée ont également été réalisées à $400^{\circ} \mathrm{C}$ sous air pendant un an soit $8736 \mathrm{~h}$.

Cette étude a pour but de comparer l'influence combinée de la microstructure et de la densité sur les propriétés de résistance à la corrosion à haute température. Il est généralement admis que le phénomène de peste, c'est-à-dire la désintégration en poudre des échantillons de $\mathrm{MoSi}_{2}$, est due à la croissance de l'oxyde de $\mathrm{Mo}\left(\mathrm{MoO}_{3}\right)$ dont l'expansion volumique entraîne la fissuration du matériau et petit à petit sa destruction. Basé sur les travaux obtenus par Chevalier et al. [17], il est possible de comparer le comportement à l'oxydation de nos matériaux avec un échantillon plus dense mais microstructuré (Figs. 7 et 9). La figure 7 montre l'évolution de la prise de masse d'un échantillon dense et nano-organisé, élaboré à partir d'un mélange de poudre de Mo et de Si activé mécaniquement et d'un échantillon dense et microstructuré, élaboré à partir d'un mélange de poudre de Mo et de 

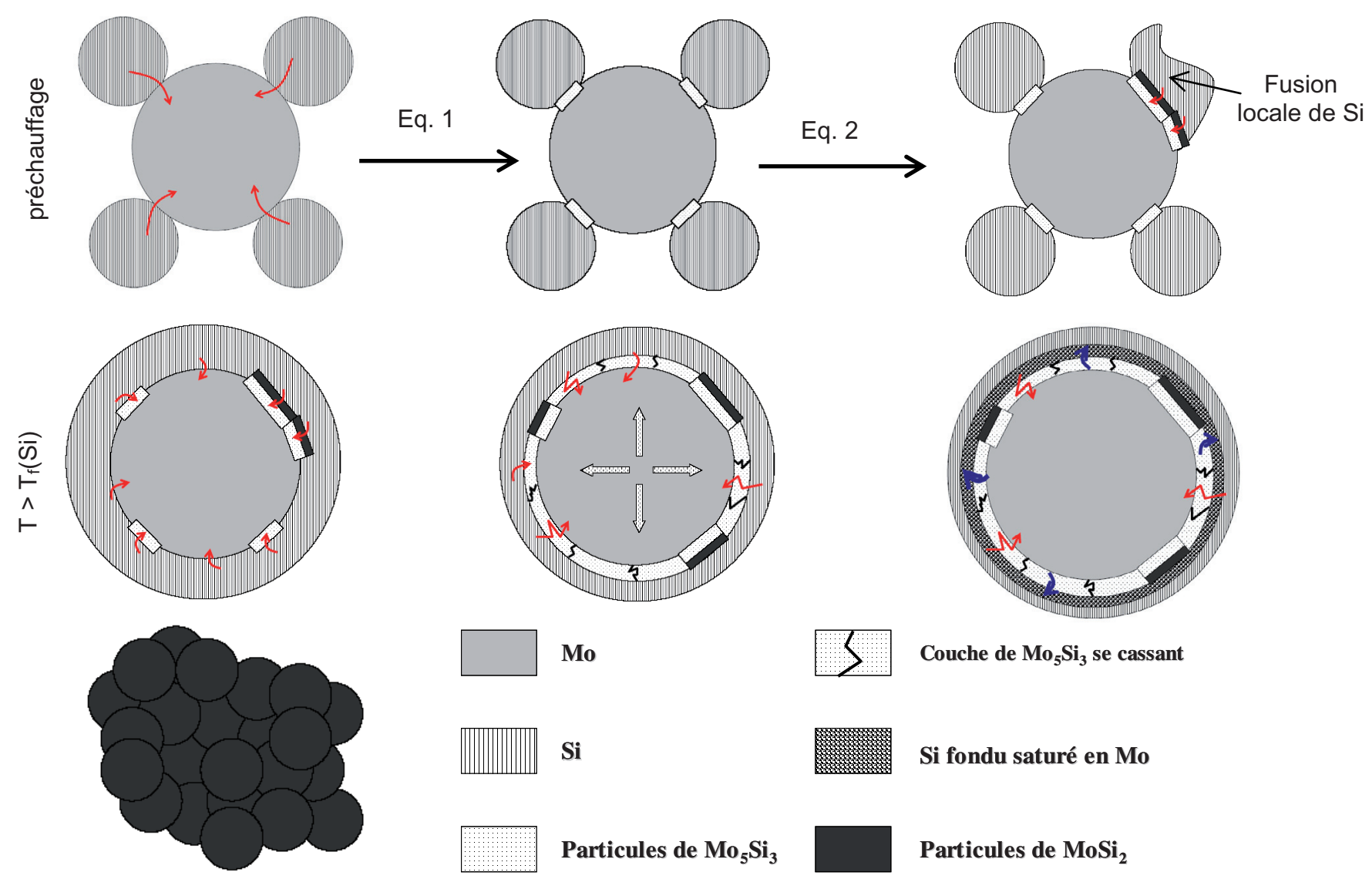

Mo

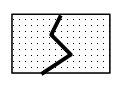

Couche de $\mathrm{Mo}_{5} \mathrm{Si}_{3}$ se cassant

Si

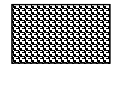

Si fondu saturé en Mo

Particules de $\mathrm{Mo}_{5} \mathrm{Si}_{3}$

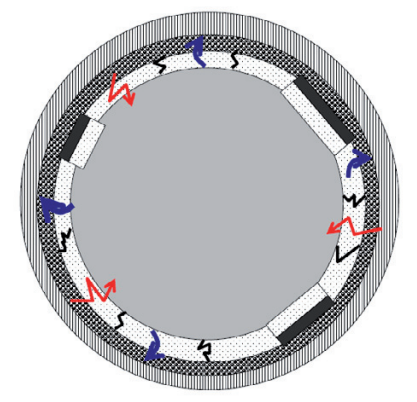

Fig. 6. Schéma du mécanisme de formation proposé de $\mathrm{MoSi}_{2}$ élaboré par frittage «flash » réactif, à partir de poudres mécaniquement activées.

Fig. 6. Proposed schematic diagram of MoSi $i_{2}$ formation mechanism during the current-assisted SHS-sintering.

Si micronique, en fonction du temps. Ces deux échantillons ont été élaborés par frittage «flash » réactif sous champ électrique. La prise de masse d'un échantillon dense nano-organisé est 10 fois plus faible que l'échantillon dense et microstructuré (Fig. 7). De plus, la valeur de constante de cinétique parabolique d'oxydation est 1000 fois plus faible que celle d'un échantillon dense et microstructuré (Fig. 7). La présence du trioxyde de molybdène peut être identifiée au niveau des pores préexistants dans le matériau de départ (Fig. 8a). Maruyama et al. [33] ont montré que les fissures et les pores préexistants dans le matériau sont principalement responsables du phénomène de peste. Malgré la présence de cet oxyde, l'observation de la surface des échantillons révèle que la majorité de la surface des échantillons est recouverte d'une couche (Fig. 8a). Les analyses EDXS indiquent que ces zones denses sont composées de Mo, de Si et de O. En couplant ces résultats avec la présence d'un halo aux faibles angles de diffraction des rayons $X$ qui suggère la présence d'une phase amorphe
(Fig. 8b), cette couche peut être identifiée comme une couche de silice amorphe. Cette couche de silice peut ralentir la croissance de l'oxyde de Mo et peut expliquer l'amélioration des propriétés de résistance à la corrosion de nos échantillons. En effet, nos échantillons conservent leur intégrité physique après une oxydation à $400^{\circ} \mathrm{C}$ sous air à la pression atmosphérique pendant 1 an (Fig. 9a) alors qu'un échantillon dense et microstructuré est réduit en poudre après une semaine à $400{ }^{\circ} \mathrm{C}$ (Fig. 9b).

L'augmentation de la densité des matériaux de départ semble être responsable du ralentissement du phénomène de peste. Néanmoins, l'ensemble de nos résultats montre que l'effet combiné de la densité et de la nano-organisation entraîne un ralentissement beaucoup plus important du phénomène de peste. Ce ralentissement pourrait être dû à la formation d'une couche de silice qui protège partiellement la surface des échantillons et qui serait rendue possible grâce à la nano-organisation du substrat $\mathrm{MoSi}_{2}$. 


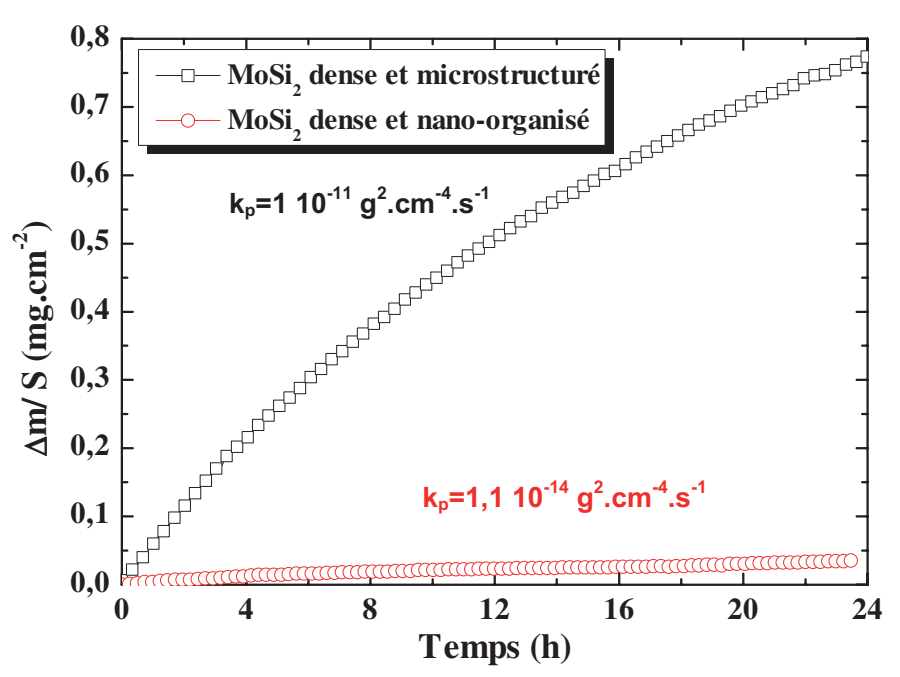

Fig. 7. Courbes de prise de masse obtenues lors de 1'oxydation sous air à $400{ }^{\circ} \mathrm{C}$ pendant $24 \mathrm{~h}$ d'un composé $\mathrm{MoSi}_{2}$ dense et nano-organisé et d'un composé $\mathrm{MoSi}_{2}$ dense et microstructuré [27].

Fig. 7. Kinetic curves of a dense nanostructured samples and a dense microstructured samples oxidized in air at $400{ }^{\circ} \mathrm{C}$ for $24 \mathrm{~h}$ (from Ref. [27]). mation dynamique de $\mathrm{MoSi}_{2}$ en utilisant la capacité du rayonnement d'un synchrotron. L'ensemble des résultats obtenu au cours de cette étude montre que la synthèse de $\mathrm{MoSi}_{2}$ par MASPS peut être divisée en six étapes :

(i) préchauffage du mélange de poudre,

(ii) nucléation de la phase secondaire $\left(\mathrm{Mo}_{5} \mathrm{Si}_{3}\right)$ à la surface du molybdène, due à une réaction de diffusion à l'état solide exothermique entre le silicium solide et le molybdène solide à partir de $500^{\circ} \mathrm{C}$,

(iii) étape $d$ 'homogénéisation, avant le point de fusion de silicium, causée par la fusion locale du silicium et une autre réaction exothermique de diffusion entre le silicium liquide et $\mathrm{Mo}_{5} \mathrm{Si}_{3}$,

(iv) les atomes de silicium mouillent les particules de Mo pour former une couche de phase intermédiaire, couche se fissurant petit à petit à cause de la dilation thermique des particules de Mo,

(v) dissolution de cette phase intermédiaire dans le silicium fondu et

(vi) formation de $\mathrm{MoSi}_{2}$ par une réaction SHS impliquant une réaction de précipitation de $\mathrm{MoSi}_{2}$ suite à la saturation du silicium en molybdène.

Néanmoins, malgré la présence de cette phase secondaire $\left(\mathrm{Mo}_{5} \mathrm{Si}_{3}\right)$, les échantillons denses et nano-organisés présentent une résistance à la corrosion améliorée. En effet, ces derniers conservent leur intégrité physique après une oxydation à $400{ }^{\circ} \mathrm{C}$ sous air pendant 1 an, alors que les échantillons denses et microstructurés sont détruits après une semaine d'oxydation à $400{ }^{\circ} \mathrm{C}$ sous air. À «basse» température, les résultats actuels indiquent que les fissures et les pores préexistants sont principalement responsables $\mathrm{du}$ phénomène de peste. Le comportement de ces composés peut être modélisé par une loi parabolique. Ce type de comportement peut être causé par la présence d'une fine couche de silice $\left(\mathrm{SiO}_{2}\right)$ qui recouvre partiellement la surface des échantillons. Cette couche a pour conséquence de ralentir la formation du trioxyde de molybdène. Cette étude indique que le ralentissement du phénomène de peste n'est pas uniquement lié à un seul paramètre. Seuls les échantillons combinant, en même temps, une microstructure nano-organisée et une densité élevée présentent un ralentissement du phénomène de peste. 

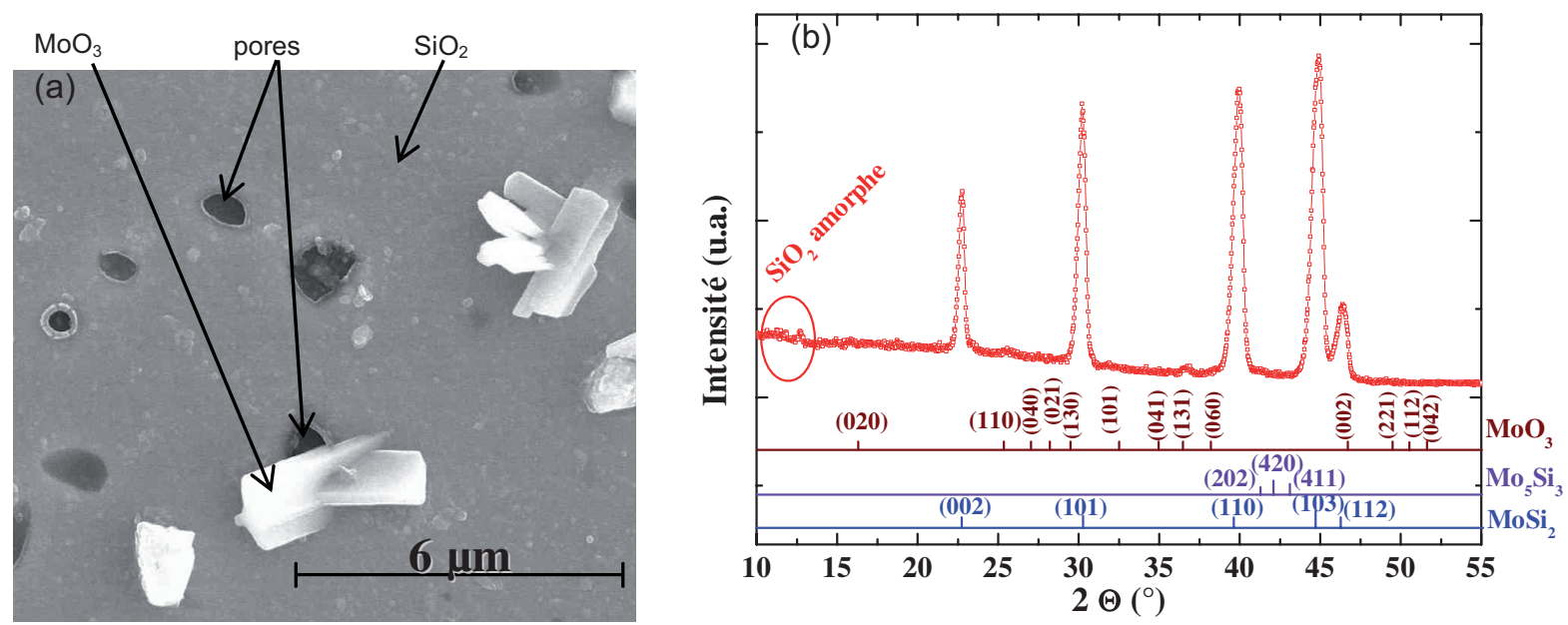

Fig. 8. (a) Micrographie MEB (électrons secondaires) du composé $\mathrm{MoSi}_{2}$ dense et nano-organisé oxydé à $400{ }^{\circ} \mathrm{C}$ sous air pendant $24 \mathrm{~h}$ : (a) observation de la couche de silice recouvrant la surface des échantillons et des aiguilles plates de $\mathrm{MoO}_{3}$ se développant dans un pore et (b) diffractogramme du composé $\mathrm{MoSi}_{2}$ dense et nano-organisé oxydé à $400{ }^{\circ} \mathrm{C}$ sous air pendant $24 \mathrm{~h}$.

Fig. 8. SEM image of dense nanostructured samples oxidized at $400{ }^{\circ} \mathrm{C}$ in synthetic air for $24 \mathrm{~h}$ : (a) SEM observation of SiO $\mathrm{S}_{2}$ scale wich covered the sample surface and (b) platelets $\mathrm{MoO}_{3}$ with grew in a pore.

(a)

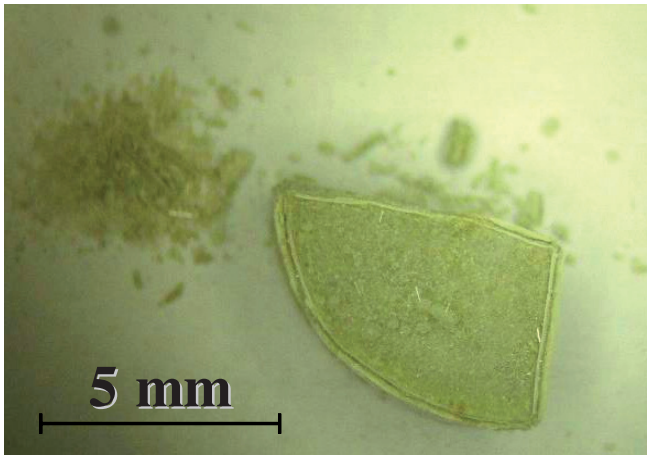

(b)

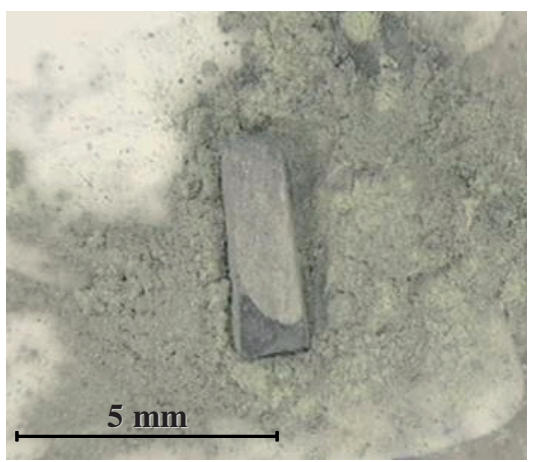

Fig. 9. Photographie de (a) un échantillon dense et nano-organisé oxydé à $400{ }^{\circ} \mathrm{C}$ pendant 52 semaines et (b) d'un échantillon dense et microstructuré oxydé à $400{ }^{\circ} \mathrm{C}$ pendant une semaine [27]. Fig. 9. Macroscopic pictures of (a) a dense nano organized MoSi $i_{2}$ oxidized 52 weeks in air and $(b)$ a dense nano organized $\mathrm{MoSi}_{2}$ oxidized 1 week in air at $400^{\circ} \mathrm{C}$ from [27].

\section{Références}

[1] I.E. Campbell, E.M. Sherwood, « Reaction synthesis of refractory disilicides by mechanical alloying and shock reactive synthesis ", High temperature Materials and Technology, Wiley, New York (1967), p. 417

[2] S.W. Jo, G.W. Lee, J.T. Moon, Y.G. Kin, Acta Mater. 44 (1996) 4317

[3] L. Xiao, Y.S. Kim, R. Abbaschian, Mater. Res. Soc. Symp. Proc. 194 (1990) 399

[4] B.K Yen, T. Aizawa, J. Kihara, Mater. Sci. Eng. A 220 (1996) 8

[5] O. Kubaschewski, C.B. Alcock, P.J. Spencer, "Materials Thermochemistry ", 6th edn., Pergamon Press, Oxford (1993)

[6] A.G. Merzhanov, I.P. Borovinska, I.O. Khomeko, A.S. Mukasyan, V.I. Ponomarev, A.S. Rogashev, V.M. Shkiro, Ann. Chim. Fr. 20 (1995) 123

[7] J.M. Lihrman, D. Vrel, J.P. Petitet, Ann. Chim. Fr. 20 (1995) 111
[8] Z.A. Munir, Ceram. Bull. 2 (1988) 342

[9] G. Fougere, J. Weertman, R. Seiger, Nanostruct. Mater. 5 (1995) 127

[10] Z.A. Munir, F. Charlot, F. Bernard, E. Gaffet, "One-Step Synthesis and Consolidation of Nanophase Materials », US Patent No. 6, 200 (2001), p. 515

[11] C. Gras, F. Bernard, F. Charlot, E. Gaffet, Z.A. Munir, J. Mater. Res. 73 (2002) 1

[12] V.Gauthier, F. Bernard, E. Gaffet, Z.A. Munir, J.P. Larpin, Intermetallics 9 (2001) 571

[13] C. Gras, D. Vrel, E. Gaffet, F. Bernard, J. Alloy. Comp. 314 (2001) 240

[14] J. Kuchino, K. Kurokawa, T. Shibayama, H. Takahashi, Vacuum 73 (2004) 623-628

[15] S. Paris, E. Gaffet, F. Bernard, Z.A. Munir, Scr. Mater. 50 (2004) 691

[16] E. Fitzer, «Warmfeste und Korrosionbestandige Sinterwerkstofffe", édité par F. Benesovsky, Pergamon Press, Elmsfor, New York (1965), p. 56 
[17] S. Chevalier, F. Bernard, E. Gaffet, S. Paris, Z.A. Munir, J.P. Larpin, Mater. Sci. Forum 461464 (2004) 439

[18] M. Abdellaoui, T. Barradi, E. Gaffet, J. Alloys Comp. 198 (1993) 155

[19] M. Omori, Mater Sci. Eng. A 287 (2000) 193

[20] Z. Shen, Z. Zhang, H. Preng, M. Nygren, Nature 417 (2002) 266

[21] Z. Shen, M. Johnsson, Z. Chao, M. Mygren, J. Am. Ceram Soc. 85 (2002) 1921

[22] G. Cabouro, S. Chevalier, E. Gaffet, Y. Grin F. Bernard, J. Alloys Comp. (2007), soumis

[23] W. Chen, U. Anselmi-Tamburini, J.E. Garay, J.R. Groza, Z.A. Munir, Mater. Sci. Eng. A 394 (2005) 132

[24] G. Cabouro, S. Chevalier, E. Gaffet, A.S. Rogachev, D. Vrel, N. Boudet, F. Bernard, Int. J. SHS (2007), accepté

[25] N. Bertino, J. Garay, U. Anselmi-Tamburini, Scr. Mater. 44 (2001) 737

[26] F. Bernard, E. Gaffet, Z.A. Munir, "Production of dense manostructured matrials usinf FAPAS and SPS techniques", dans Pulse Electric Current Synthesis and Processing Metarials : Ceramic Transition, édité par Z.A. Munir, M. Ohyanagi, M. Tokita, M. Khor, T. Hirai, U. AnselmiTamburini, Vol. 194 (2006)

[27] J. Gao, W. Jiang, G. Wang, Key Eng. Mater. 280-283 (2005) 1467

[28] V.V. Aleksandrov, M.A. Korchagin, Explos. Shock Waves USSR 5 (1987) 557

[29] A. Biswas, S.K. Roy, Acta Mater. 52 (2004) 257

[30] S.C. Deevi, Mater. Sci. Eng. A 142 (1992) 241

[31] L.S. Darken, R.W. Gurry, Physical Chemistry of Metals, dans Metallurgy and Metallurgical Engineering Series, McGraw-Hill, New York (1953)

[32] N. Birks, G.H. Meier, Introduction to high temperature oxidation of metals, Edwards Arnold, London (1983), p. 42

[33] T. Maruyama, K. Yanagihara, Mater. Sci. Eng. A 239-240 (1997) 828 